\title{
On asymptotically strict pseudocontractions and equilibrium problems
}

Peng Cheng ${ }^{1}$ and Huilian $\mathrm{Wu}^{2^{*}}$

"Correspondence: hbwuhl@yeah.net

${ }^{2}$ College of Science, Hebei

University of Engineering, Handan,

056038, China

Full list of author information is

available at the end of the article

\begin{abstract}
In this paper, an equilibrium problem and a fixed point problem of an asymptotically strict pseudocontraction are investigated based on hybrid iterative algorithms. Strong convergence theorems of common solutions to the equilibrium problem and the fixed point problem are obtained in the framework of real Hilbert spaces.
\end{abstract}

MSC: $47 \mathrm{H} 09 ; 47 \mathrm{H} 10$

Keywords: asymptotically strict pseudocontraction; asymptotically nonexpansive mapping; nonexpansive mapping; fixed point; equilibrium problem

\section{Introduction}

Equilibrium problems have emerged as an effective and powerful tool for studying a wide class of problems which arise in economics, finance, image reconstruction, ecology, transportation, and network; see [1-12] and the references therein. Equilibrium problems include fixed point problems, variational inequality problems, variational inclusion problems, saddle point problems, the Nash equilibrium problem, complementarity problems and so on. For the solutions of equilibrium problems, there are several algorithms to solve the problem. The classical algorithm is the Krasnoselskii-Mann iterative algorithm. However, the Krasnoselskii-Mann iterative algorithm is weak convergence for the solutions of equilibrium problems. Haugazeau's projection method [13] recently has been considered for the approximation of solutions of equilibrium problems and fixed point problems. The advantage of the projection methods is that strong convergence of iterative sequences can be guaranteed without any compact assumptions. The aim of this paper is to study an equilibrium problem and a fixed point problem of an asymptotically strict pseudocontraction based on hybrid iterative algorithms and establish a strong convergence theorem of common solutions in the framework of Hilbert spaces.

\section{Preliminaries}

Throughout this paper, we always assume that $H$ is a real Hilbert space with the inner product $\langle\cdot, \cdot\rangle$ and the norm $\|\cdot\|$. Let $C$ be a nonempty closed convex subset of $H$, let $A: C \rightarrow H$ be a monotone mapping and $F$ be a bifunction of $C \times C$ into $\mathbb{R}$, where $\mathbb{R}$ denotes the set of real numbers.

In this paper, we consider the following equilibrium problem.

$$
\text { Find } x \in C \text { such that } F(x, y)+\langle A x, y-x\rangle \geq 0, \quad \forall y \in C \text {. }
$$

(C) 2013 Cheng and Wu; licensee Springer. This is an Open Access article distributed under the terms of the Creative Commons Attribution License (http://creativecommons.org/licenses/by/2.0), which permits unrestricted use, distribution, and reproduction in any medium, provided the original work is properly cited. 
The set of such an $x \in C$ is denoted by $\operatorname{EP}(F, A)$, i.e.,

$$
\operatorname{EP}(F, A)=\{x \in C: F(x, y)+\langle A x, y-x\rangle \geq 0, \forall y \in C\}
$$

If $A \equiv 0$, then the problem (2.1) is reduced to the following:

$$
\text { Find } x \in C \text { such that } F(x, y) \geq 0, \quad \forall y \in C \text {. }
$$

The set of such an $x \in C$ is denoted by $\operatorname{EP}(F)$, i.e.,

$$
\mathrm{EP}(F, A)=\{x \in C: F(x, y) \geq 0, \forall y \in C\} .
$$

If $F \equiv 0$, the problem (2.1) is reduced to the classical variational inequality problem.

Find $x \in C$ such that $\langle A x, y-x\rangle \geq 0, \quad \forall y \in C$.

To study the problems (2.1) and (2.2), we may assume that $F$ satisfies the following conditions:

(A1) $F(x, x)=0$ for all $x \in C$;

(A2) $F$ is monotone, i.e., $F(x, y)+F(y, x) \leq 0$ for all $x, y \in C$;

(A3) for each $x, y, z \in C$,

$$
\limsup _{t \downarrow 0} F(t z+(1-t) x, y) \leq F(x, y)
$$

(A4) for each $x \in C, y \mapsto F(x, y)$ is convex and lower semi-continuous.

Recall that a mapping $A$ is said to be monotone iff

$$
\langle A x-A y, x-y\rangle \geq 0, \quad \forall x, y \in C
$$

$A$ is said to be strongly monotone iff there exists a constant $\alpha>0$ such that

$$
\langle A x-A y, x-y\rangle \geq \alpha\|x-y\|^{2}, \quad \forall x, y \in C .
$$

For such a case, $A$ is said to be $\alpha$-strongly-monotone. $A$ is said to be inverse-strongly monotone iff there exists a constant $\alpha>0$ such that

$$
\langle A x-A y, x-y\rangle \geq \alpha\|A x-A y\|^{2}, \quad \forall x, y \in C .
$$

For such a case, $A$ is said to be $\alpha$-inverse-strongly monotone.

Recall that a set-valued mapping $T: H \rightarrow 2^{H}$ is said to be monotone iff, for all $x, y \in H$, $f \in T x$ and $g \in T y$ imply $\langle x-y, f-g\rangle \geq 0 . T: H \rightarrow 2^{H}$ is maximal if the graph $G(T)$ of $T$ is not properly contained in the graph of any other monotone mapping.

It is known that a monotone mapping $T$ is maximal if and only if, for any $(x, f) \in H \times H$, $\langle x-y, f-g\rangle \geq 0$ for all $(y, g) \in G(T)$ implies $f \in T x$.

Let $S: C \rightarrow C$ be a nonlinear mapping. In this paper, we use $F(S)$ to denote the fixed point set of $S$. 
Recall that $S$ is said to be nonexpansive iff

$$
\|S x-S y\| \leq\|x-y\|, \quad \forall x, y \in C .
$$

Recall that $S$ is said to be asymptotically nonexpansive iff there exists a sequence $\left\{k_{n}\right\} \subset$ $[1, \infty)$ with $k_{n} \rightarrow 1$ as $n \rightarrow \infty$ such that

$$
\left\|S^{n} x-S^{n} y\right\| \leq k_{n}\|x-y\|, \quad \forall x, y \in C, n \geq 1 .
$$

The class of asymptotically nonexpansive mappings was introduced by Goebel and Kirk [14] in 1972. Since 1972, a number of authors have studied the convergence problems of the iterative processes for such a class of mappings.

Recall that $S$ is said to be strictly pseudocontractive iff there exists a constant $\kappa \in[0,1)$ such that

$$
\|S x-S y\|^{2} \leq\|x-y\|^{2}+\kappa\|(I-S) x-(I-S) y\|^{2}, \quad \forall x, y \in C .
$$

For such a case, $S$ is also said to be a $\kappa$-strict pseudocontraction. The class of strict pseudocontractions is introduced by Browder and Petryshyn [15] in 1967. It is clear that every nonexpansive mapping is a 0 -strict pseudocontraction. We also remark that if $\kappa=1$, then $S$ is said to be pseudocontractive.

Recall that $S$ is said to be an asymptotically strict pseudocontraction iff there exist a sequence $\left\{k_{n}\right\} \subset[1, \infty)$ with $k_{n} \rightarrow 1$ as $n \rightarrow \infty$ and a constant $\kappa \in[0,1)$ such that

$$
\left\|S^{n} x-S^{n} y\right\|^{2} \leq k_{n}\|x-y\|^{2}+\kappa\left\|\left(I-S^{n}\right) x-\left(I-S^{n}\right) y\right\|^{2}, \quad \forall x, y \in C, n \geq 1 .
$$

For such a case, $S$ is also said to be an asymptotically $\kappa$-strict pseudocontraction. The class of asymptotically strict pseudocontractions is introduced by Qihou [16] in 1996. It is clear that every asymptotically nonexpansive mapping is an asymptotical 0 -strict pseudocontraction. Every nonexpansive mapping is an asymptotically nonexpansive mapping with the sequence $\{1\}$. We also remark that if $\kappa=1$, then $S$ is said to be an asymptotically pseudocontractive mapping which was introduced by Schu [17] in 1991.

Recently, many authors considered the equilibrium problems (2.1), (2.2) and fixed point problems based on hybrid iterative methods; see, for instance, [18-30]. In this paper, motivated by these recent results, we consider the shrinking projection algorithm to solve the solutions of the equilibrium problem (2.1) and the fixed point problem of an asymptotically strict pseudocontraction. It is proved that the sequence generated in the purposed iterative process converges strongly to some common element in the solution set of the equilibrium problem (2.1) and in the fixed point set of an asymptotically strict pseudocontraction.

In order to prove our main results, we also need the following lemmas.

Lemma 2.1 [31] Let $C$ be a nonempty closed convex subset of $H$ and let $F: C \times C \rightarrow \mathbb{R}$ be a bifunction satisfying (A1)-(A4). Then, for any $r>0$ and $x \in H$, there exists $z \in C$ such that

$$
F(z, y)+\frac{1}{r}\langle y-z, z-x\rangle \geq 0, \quad \forall y \in C .
$$


Further, define

$$
T_{r} x=\left\{z \in C: F(z, y)+\frac{1}{r}\langle y-z, z-x\rangle \geq 0, \forall y \in C\right\}
$$

for all $r>0$ and $x \in H$. Then the following hold:

(a) $T_{r}$ is single-valued;

(b) $T_{r}$ is firmly nonexpansive, i.e., for any $x, y \in H$,

$$
\left\|T_{r} x-T_{r} y\right\|^{2} \leq\left\langle T_{r} x-T_{r} y, x-y\right\rangle
$$

(c) $F\left(T_{r}\right)=\mathrm{EP}(F)$;

(d) $\mathrm{EP}(F)$ is closed and convex.

Lemma 2.2 [32] In a real Hilbert space, the following inequality holds:

$$
\|a x+(1-a) y\|^{2}=a\|x\|^{2}+(1-a)\|y\|^{2}-a(1-a)\|x-y\|^{2}, \quad \forall a \in[0,1], x, y \in H .
$$

Lemma 2.3 [33] Let $H$ be a real Hilbert space, let $C$ be a nonempty closed convex subset of $H$ and let $S: C \rightarrow C$ be an asymptotically $\kappa$-strict pseudocontraction with the sequence $\left\{k_{n}\right\}$. Then

(a) $F(S)$ is closed and convex;

(b) $S$ is L-Lipschitz continuous.

Lemma 2.4 [33] Let $H$ be a real Hilbert space, let $C$ be a nonempty closed convex subset of $H$ and let $S: C \rightarrow C$ be an asymptotically strict pseudocontraction. Then the mapping $I-S$ is demiclosed at zero, that is, if $\left\{x_{n}\right\}$ is a sequence in C such that $x_{n}-\bar{x}$ and $x_{n}-S x_{n} \rightarrow 0$, then $\bar{x} \in F(S)$.

\section{Main results}

Theorem 3.1 Let $C$ be a nonempty closed convex subset of a real Hilbert space $H$. Let $F_{m}$ be a bifunction from $C \times C$ to $\mathbb{R}$ which satisfies (A1)-(A4) and let $A_{m}: C \rightarrow H$ be a $\xi_{m}$-inverse-strongly monotone mapping for each $1 \leq m \leq N$, where $N \geq 1$ is some positive integer. Let $S: C \rightarrow C$ be an asymptotically $\kappa$-strict pseudocontraction. Assume that $\mathcal{F}:=$ $F(S) \cap \bigcap_{m=1}^{N} \operatorname{EP}\left(F_{m}, A_{m}\right)$ is nonempty and bounded. Let $\left\{\alpha_{n}\right\}$ and $\left\{\beta_{n}\right\}$ be sequences in $[0,1]$ and let $\left\{r_{n, m}\right\}$ be a positive sequence. Let $\left\{\gamma_{n, m}\right\}$ be a sequence in $[0,1]$ for each $1 \leq m \leq N$ such that $\sum_{m=1}^{N} \gamma_{n, m}=1$. Let $\left\{x_{n}\right\}$ be a sequence generated in the following manner:

$$
\left\{\begin{array}{l}
x_{1} \in H, \\
C_{1}=H, \\
F_{m}\left(u_{n, m}, u\right)+\left\langle A_{m} x_{n}, u-u_{n, m}\right\rangle+\frac{1}{r_{n, m}}\left\langle u-u_{n, m}, u_{n, m}-x_{n}\right\rangle \geq 0, \quad \forall u \in C, \\
y_{n}=\alpha_{n} x_{n}+\left(1-\alpha_{n}\right)\left(\beta_{n} \sum_{m=1}^{N} \gamma_{n, m} u_{n, m}+\left(1-\beta_{n}\right) S^{n} \sum_{m=1}^{N} \gamma_{n, m} u_{n, m}\right), \\
C_{n+1}=\left\{w \in C_{n}:\left\|y_{n}-w\right\|^{2} \leq\left\|x_{n}-w\right\|^{2}+\theta_{n}\right\}, \\
x_{n+1}=P_{C_{n+1}} x_{1}, \quad n \geq 0,
\end{array}\right.
$$

where $\theta_{n}=\left(1-\alpha_{n}\right)\left(k_{n}-1\right) \Theta_{n}$ and $\Theta_{n}=\sup \left\{\left\|x_{n}-w\right\|^{2}: w \in \mathcal{F}\right\}$. Assume that the control sequences $\left\{\alpha_{n}\right\},\left\{\beta_{n}\right\},\left\{\gamma_{n, 1}\right\}, \ldots,\left\{\gamma_{n, N}\right\},\left\{r_{n, 1}\right\}, \ldots$, and $\left\{r_{n, N}\right\}$ satisfy the following restrictions: 
(a) $0 \leq \alpha_{n} \leq a<1, \kappa \leq \beta_{n} \leq b<1$;

(b) $0<c \leq \gamma_{n, m} \leq 1$ and $0<d \leq r_{n, m} \leq e<2 \xi_{m}$ for each $1 \leq m \leq N$.

Then the sequence $\left\{x_{n}\right\}$ converges strongly to some point $\bar{x}$, where $\bar{x}=P_{\mathcal{F}} x_{1}$.

Proof First, we show that $C_{n}$ is closed and convex for each $n \geq 1$. It is easy to see that $C_{n}$ is closed for each $n \geq 1$. We only show that $C_{n}$ is convex for each $n \geq 1$. Note that $C_{1}=H$ is convex. Suppose that $C_{i}$ is convex for some positive integer $i$. Next, we show that $C_{i+1}$ is convex for the same $i$. Note that

$$
\left\|y_{i}-w\right\|^{2} \leq\left\|x_{i}-w\right\|^{2}+\theta_{i}
$$

is equivalent to

$$
2\left\langle x_{i}-y_{i}, w\right\rangle \leq\left\|x_{i}\right\|^{2}-\left\|y_{i}\right\|^{2}+\theta_{i}
$$

Take $w_{1}$ and $w_{2}$ in $C_{i+1}$ and put $\bar{w}=t w_{1}+(1-t) w_{2}$. It follows that $w_{1} \in C_{i}, w_{2} \in C_{i}$,

$$
2\left\langle x_{i}-y_{i}, w_{1}\right\rangle \leq\left\|x_{i}\right\|^{2}-\left\|y_{i}\right\|^{2}+\theta_{i}
$$

and

$$
2\left\langle x_{i}-y_{i}, w_{2}\right\rangle \leq\left\|x_{i}\right\|^{2}-\left\|y_{i}\right\|^{2}+\theta_{i} .
$$

Combining (3.2) with (3.3), we can obtain that $2\left\langle x_{i}-y_{i}, \bar{w}\right\rangle \leq\left\|x_{i}\right\|^{2}-\left\|y_{i}\right\|^{2}+\theta_{i}$, that is, $\left\|y_{i}-w\right\|^{2} \leq\left\|x_{i}-\bar{x}\right\|^{2}+\theta_{i}$. In view of the convexity of $C_{i}$, we see that $\bar{w} \in C_{i}$. This shows that $\bar{w} \in C_{i+1}$. This concludes that $C_{n}$ is closed and convex for each $n \geq 1$. Notice that $\left(I-r_{n, m} A_{m}\right)$ is nonexpansive. Indeed, for any $x, y \in C$, we find from the restriction (b) that

$$
\begin{aligned}
\| & \left(I-r_{n, m} A_{m}\right) x-\left(I-r_{n, m} A_{m}\right) y \|^{2} \\
& =\left\|(x-y)-r_{n, m}\left(A_{m} x-A_{m} y\right)\right\|^{2} \\
& =\|x-y\|^{2}-2 r_{n, m}\left\langle x-y, A_{m} x-A_{m} y\right\rangle+r_{n, m}^{2}\left\|A_{m} x-A_{m} y\right\|^{2} \\
& \leq\|x-y\|^{2}-2 r_{n, m} \xi_{m}\left\|A_{m} x-A_{m} y\right\|^{2}+r_{n, m}^{2}\left\|A_{m} x-A_{m} y\right\|^{2} \\
& =\|x-y\|^{2}+r_{n, m}\left(r_{n, m}-2 \xi_{m}\right)\left\|A_{m} x-A_{m} y\right\|^{2} \\
& \leq\|x-y\|^{2} .
\end{aligned}
$$

It follows that

$$
\begin{aligned}
\left\|u_{n, m}-p\right\|^{2} & =\left\|T_{r_{n, m}}\left(x_{n}-r_{n, m} A_{m} x_{n}\right)-T_{r_{n, m}}\left(p-r_{n, m} A_{m} p\right)\right\|^{2} \\
& \leq\left\|\left(x_{n}-r_{n, m} A_{m} x_{n}\right)-\left(p-r_{n, m} A_{m} p\right)\right\|^{2} \\
& \leq\left\|x_{n}-p\right\|^{2} .
\end{aligned}
$$

Put $z_{n}=\sum_{m=1}^{N} \gamma_{n, m} u_{n, m}$ for each $n \geq 1$. Next, we show that $\mathcal{F} \subset C_{n}$ for all $n \geq 1$. It is easy to see that $\mathcal{F} \subset C_{1}=H$. Suppose that $\mathcal{F} \subset C_{h}$ for some integer $h \geq 1$. We intend to claim that 
$\mathcal{F} \subset C_{h+1}$ for the same $h$. For any $p \in \mathcal{F} \subset C_{h}$, we have from Lemma 2.2 and the restriction (a) that

$$
\begin{aligned}
\left\|y_{h}-p\right\|^{2} & \left\|\alpha_{h} x_{h}+\left(1-\alpha_{h}\right)\left(\beta_{h} z_{h}+\left(1-\beta_{h}\right) S^{h} z_{h}\right)-p\right\|^{2} \\
\leq & \alpha_{h}\left\|x_{h}-p\right\|^{2}+\left(1-\alpha_{h}\right)\left\|\beta_{h}\left(z_{h}-p\right)+\left(1-\beta_{h}\right)\left(S^{h} z_{h}-S^{h} p\right)\right\|^{2} \\
= & \alpha_{h}\left\|x_{h}-p\right\|^{2}+\left(1-\alpha_{h}\right)\left(\beta_{h}\left\|z_{h}-p\right\|^{2}+\left(1-\beta_{h}\right)\left\|S^{h} z_{h}-S^{h} p\right\|^{2}\right. \\
& \left.\quad-\beta_{h}\left(1-\beta_{h}\right)\left\|z_{h}-p-\left(S^{h} z_{h}-S^{h} p\right)\right\|^{2}\right) \\
\leq & \alpha_{h}\left\|x_{h}-p\right\|^{2}+\left(1-\alpha_{h}\right) \beta_{h}\left\|z_{h}-p\right\|^{2}+\left(1-\alpha_{h}\right)\left(1-\beta_{h}\right)\left(k_{h}\left\|z_{h}-p\right\|^{2}\right. \\
& \left.+\kappa\left\|z_{h}-p-\left(S^{h} z_{h}-S^{h} p\right)\right\|^{2}\right)-\left(1-\alpha_{h}\right) \beta_{h}\left(1-\beta_{h}\right)\left\|z_{h}-p-\left(S^{h} z_{h}-S^{h} p\right)\right\|^{2} \\
\leq & \alpha_{h}\left\|x_{h}-p\right\|^{2}+\left(1-\alpha_{h}\right) k_{h}\left\|z_{h}-p\right\|^{2} \\
& \quad-\left(1-\alpha_{h}\right)\left(1-\beta_{h}\right)\left(\beta_{h}-\kappa\right)\left\|z_{h}-p-\left(S^{h} z_{h}-S^{h} p\right)\right\|^{2} \\
\leq & \alpha_{h}\left\|x_{h}-p\right\|^{2}+\left(1-\alpha_{h}\right) k_{h}\left\|z_{h}-p\right\|^{2} \\
\leq & \alpha_{h}\left\|x_{h}-p\right\|^{2}+\left(1-\alpha_{h}\right) k_{h} \sum_{m=1}^{N} \gamma_{h, m}\left\|T_{r_{h, m}}\left(x_{h}-r_{h, m} A_{m} x_{h}\right)-p\right\|^{2} \\
\leq & \alpha_{h}\left\|x_{h}-p\right\|^{2}+\left(1-\alpha_{h}\right) k_{h}\left\|x_{h}-p\right\|^{2} \\
\leq & \left\|x_{h}-p\right\|^{2}+\theta_{h} .
\end{aligned}
$$

This shows that $p \in C_{h+1}$. This proves that $\mathcal{F} \subset C_{n}$ for all $n \geq 1$. Since $x_{n}=P_{C_{n}} x_{1}$ and $x_{n+1}=P_{C_{n+1}} x_{1} \in C_{n+1} \subset C_{n}$, we have that

$$
\begin{aligned}
0 & \leq\left\langle x_{1}-x_{n}, x_{n}-x_{n+1}\right\rangle \\
& =\left\langle x_{1}-x_{n}, x_{n}-x_{1}+x_{1}-x_{n+1}\right\rangle \\
& \leq-\left\|x_{1}-x_{n}\right\|^{2}+\left\|x_{1}-x_{n}\right\|\left\|x_{1}-x_{n+1}\right\| .
\end{aligned}
$$

It follows that

$$
\left\|x_{1}-x_{n}\right\| \leq\left\|x_{1}-x_{n+1}\right\|
$$

On the other hand, for any $p \in \mathcal{F} \subset C_{n}$, we see that $\left\|x_{1}-x_{n}\right\| \leq\left\|x_{1}-p\right\|$. In particular, we have

$$
\left\|x_{1}-x_{n}\right\| \leq\left\|x_{1}-P_{\mathcal{F}} x_{1}\right\|
$$

This shows that the sequence $\left\{x_{n}\right\}$ is bounded. In view of (3.6), we see that $\lim _{n \rightarrow \infty}\left\|x_{n}-x_{1}\right\|$ exists. It follows from (3.5) that

$$
\begin{aligned}
& \left\|x_{n}-x_{n+1}\right\|^{2} \\
& \quad=\left\|x_{n}-x_{1}+x_{1}-x_{n+1}\right\|^{2}
\end{aligned}
$$




$$
\begin{aligned}
& =\left\|x_{n}-x_{1}\right\|^{2}+2\left\langle x_{n}-x_{1}, x_{1}-x_{n+1}\right\rangle+\left\|x_{1}-x_{n+1}\right\|^{2} \\
& =\left\|x_{n}-x_{1}\right\|^{2}+2\left\langle x_{n}-x_{1}, x_{1}-x_{n}+x_{n}-x_{n+1}\right\rangle+\left\|x_{1}-x_{n+1}\right\|^{2} \\
& =\left\|x_{n}-x_{1}\right\|^{2}-2\left\|x_{n}-x_{1}\right\|^{2}+2\left\langle x_{n}-x_{1}, x_{n}-x_{n+1}\right\rangle+\left\|x_{1}-x_{n+1}\right\|^{2} \\
& \leq\left\|x_{1}-x_{n+1}\right\|^{2}-\left\|x_{n}-x_{1}\right\|^{2}
\end{aligned}
$$

which implies that

$$
\lim _{n \rightarrow \infty}\left\|x_{n}-x_{n+1}\right\|=0
$$

In view of $x_{n+1}=P_{C_{n+1}} x_{1} \in C_{n+1}$, we find that

$$
\left\|y_{n}-x_{n+1}\right\|^{2} \leq\left\|x_{n}-x_{n+1}\right\|^{2}+\theta_{n} .
$$

This combines with (3.7) yielding that

$$
\lim _{n \rightarrow \infty}\left\|y_{n}-x_{n+1}\right\|=0
$$

Notice that $\left\|y_{n}-x_{n}\right\| \leq\left\|y_{n}-x_{n+1}\right\|+\left\|x_{n+1}-x_{n}\right\|$. Combining (3.7) with (3.8), we find that

$$
\lim _{n \rightarrow \infty}\left\|x_{n}-y_{n}\right\|=0
$$

Note that

$$
\begin{aligned}
\left\|x_{n}-y_{n}\right\| & =\left\|x_{n}-\alpha_{n} x_{n}-\left(1-\alpha_{n}\right)\left(\beta_{n} z_{n}+\left(1-\beta_{n}\right) S^{n} z_{n}\right)\right\| \\
& =\left(1-\alpha_{n}\right)\left\|x_{n}-\left(\beta_{n} z_{n}+\left(1-\beta_{n}\right) S^{n} z_{n}\right)\right\| .
\end{aligned}
$$

In view of the restriction (a), we obtain from (3.9) that

$$
\lim _{n \rightarrow \infty}\left\|x_{n}-\left(\beta_{n} z_{n}+\left(1-\beta_{n}\right) S^{n} z_{n}\right)\right\|=0 .
$$

Notice that

$$
\begin{aligned}
\left\|u_{n, m}-p\right\|^{2}= & \left\|T_{r_{n, m}}\left(I-r_{n, m} A_{m}\right) x_{n}-T_{r_{n, m}}\left(I-r_{n, m} A_{m}\right) p\right\|^{2} \\
\leq & \left\langle\left(I-r_{n, m} A_{m}\right) x_{n}-\left(I-r_{n, m} A_{m}\right) p, u_{n, m}-p\right\rangle \\
= & \frac{1}{2}\left(\left\|\left(I-r_{n, m} A_{m}\right) x_{n}-\left(I-r_{n, m} A_{m}\right) p\right\|^{2}+\left\|u_{n, m}-p\right\|^{2}\right. \\
& \left.-\left\|\left(I-r_{n, m} A_{m}\right) x_{n}-\left(I-r_{n, m} A_{m}\right) p-\left(u_{n, m}-p\right)\right\|^{2}\right) \\
\leq & \frac{1}{2}\left(\left\|x_{n}-p\right\|^{2}+\left\|u_{n, m}-p\right\|^{2}-\left\|\left(x_{n}-u_{n, m}\right)-r_{n, m}\left(A_{m} x_{n}-A_{m} p\right)\right\|^{2}\right) \\
= & \frac{1}{2}\left(\left\|x_{n}-p\right\|^{2}+\left\|u_{n, m}-p\right\|^{2}-\left\|x_{n}-u_{n, m}\right\|^{2}\right. \\
& \left.+2 r_{n, m}\left\langle x_{n}-u_{n, m}, A_{m} x_{n}-A_{m} p\right\rangle-r_{n, m}^{2}\left\|A_{m} x_{n}-A_{m} p\right\|^{2}\right)
\end{aligned}
$$


and hence

$$
\begin{aligned}
& \left\|u_{n, m}-p\right\|^{2} \\
& \quad \leq\left\|x_{n}-p\right\|^{2}-\left\|x_{n}-u_{n, m}\right\|^{2}+2 r_{n, m}\left\langle x_{n}-u_{n, m}, A_{m} x_{n}-A_{m} p\right\rangle-r_{n, m}^{2}\left\|A_{m} x_{n}-A_{m} p\right\|^{2} \\
& \quad \leq\left\|x_{n}-p\right\|^{2}-\left\|x_{n}-u_{n, m}\right\|^{2}+2 r_{n, m}\left\|x_{n}-u_{n, m}\right\|\left\|A_{m} x_{n}-A_{m}\right\| .
\end{aligned}
$$

It follows from Lemma 2.2 and the restriction (a) that

$$
\begin{aligned}
\left\|y_{n}-p\right\|^{2} & \left\|\alpha_{n} x_{n}+\left(1-\alpha_{n}\right)\left(\beta_{n} z_{n}+\left(1-\beta_{n}\right) S^{n} z_{n}\right)-p\right\|^{2} \\
\leq & \alpha_{n}\left\|x_{n}-p\right\|^{2}+\left(1-\alpha_{n}\right)\left\|\beta_{n}\left(z_{n}-p\right)+\left(1-\beta_{n}\right)\left(S^{n} z_{n}-S^{n} p\right)\right\|^{2} \\
= & \alpha_{n}\left\|x_{n}-p\right\|^{2}+\left(1-\alpha_{n}\right)\left(\beta_{n}\left\|z_{n}-p\right\|^{2}+\left(1-\beta_{n}\right)\left\|S^{n} z_{n}-S^{n} p\right\|^{2}\right. \\
& \left.\quad-\beta_{n}\left(1-\beta_{n}\right)\left\|z_{n}-p-\left(S^{n} z_{n}-S^{n} p\right)\right\|^{2}\right) \\
\leq & \alpha_{n}\left\|x_{n}-p\right\|^{2}+\left(1-\alpha_{n}\right) \beta_{n}\left\|z_{n}-p\right\|^{2}+\left(1-\alpha_{n}\right)\left(1-\beta_{n}\right)\left(k_{n}\left\|z_{n}-p\right\|^{2}\right. \\
& \left.+\kappa\left\|z_{n}-p-\left(S^{n} z_{n}-S^{n} p\right)\right\|^{2}\right)-\left(1-\alpha_{n}\right) \beta_{n}\left(1-\beta_{n}\right)\left\|z_{n}-p-\left(S^{n} z_{n}-S^{n} p\right)\right\|^{2} \\
\leq & \alpha_{n}\left\|x_{n}-p\right\|^{2}+\left(1-\alpha_{n}\right) k_{n}\left\|z_{n}-p\right\|^{2} \\
& \quad-\left(1-\alpha_{n}\right)\left(1-\beta_{n}\right)\left(\beta_{n}-\kappa\right)\left\|z_{n}-p-\left(S^{n} z_{n}-S^{n} p\right)\right\|^{2} \\
\leq & \alpha_{n}\left\|x_{n}-p\right\|^{2}+\left(1-\alpha_{n}\right) k_{n}\left\|z_{n}-p\right\|^{2} .
\end{aligned}
$$

It follows that

$$
\begin{aligned}
\left\|y_{n}-p\right\|^{2} \leq & \left(1-\alpha_{n}\right) k_{n} \sum_{m=1}^{N} \gamma_{n, m}\left\|T_{r_{n, m}}\left(x_{n}-r_{n, m} A_{m} x_{n}\right)-T_{r_{n, m}}\left(p-r_{n, m} A_{m} p\right)\right\|^{2} \\
& +\alpha_{n}\left\|x_{n}-p\right\|^{2} \\
\leq & \alpha_{n}\left\|x_{n}-p\right\|^{2}+\left(1-\alpha_{n}\right) k_{n} \sum_{m=1}^{N} \gamma_{n, m}\left\|\left(x_{n}-r_{n, m} A_{m} x_{n}\right)-\left(p-r_{n, m} A_{m} p\right)\right\|^{2} \\
\leq & \left\|x_{n}-p\right\|^{2}+\left(1-\alpha_{n}\right)\left(k_{n}-1\right)\left\|x_{n}-p\right\|^{2} \\
& \quad-r_{n, m}\left(2 \xi_{m}-r_{n, m}\right)\left(1-\alpha_{n}\right) k_{n} \sum_{m=1}^{N} \gamma_{n, m}\left\|A_{m} x_{n}-A_{m} p\right\|^{2} .
\end{aligned}
$$

This implies that

$$
\begin{aligned}
& r_{n, m}\left(2 \xi_{m}-r_{n, m}\right)\left(1-\alpha_{n}\right) k_{n} \gamma_{n, m}\left\|A_{m} x_{n}-A_{m} p\right\|^{2} \\
& \quad \leq\left\|x_{n}-p\right\|^{2}-\left\|y_{n}-p\right\|^{2}+\left(1-\alpha_{n}\right)\left(k_{n}-1\right)\left\|x_{n}-p\right\|^{2} \\
& \quad \leq\left(\left\|x_{n}-p\right\|+\left\|y_{n}-p\right\|\right)\left\|x_{n}-y_{n}\right\|+\left(1-\alpha_{n}\right)\left(k_{n}-1\right)\left\|x_{n}-p\right\|^{2} .
\end{aligned}
$$

In view of the restrictions (a) and (b), we find from (3.9) that

$$
\lim _{n \rightarrow \infty}\left\|A_{m} x_{n}-A_{m} p\right\|=0
$$


On the other hand, we find from (3.11) and (3.12) that

$$
\begin{aligned}
\left\|y_{n}-p\right\|^{2} \leq & \alpha_{n}\left\|x_{n}-p\right\|^{2}+\left(1-\alpha_{n}\right) k_{n} \sum_{m=1}^{N} \gamma_{n, m}\left\|u_{n, m}-p\right\|^{2} \\
\leq & \left\|x_{n}-p\right\|^{2}+\left(1-\alpha_{n}\right)\left(k_{n}-1\right)\left\|x_{n}-p\right\|^{2}-\left(1-\alpha_{n}\right) k_{n} \sum_{m=1}^{N} \gamma_{n, m}\left\|x_{n}-u_{n, m}\right\|^{2} \\
& +2 r_{n, m}\left(1-\alpha_{n}\right) k_{n} \sum_{m=1}^{N} \gamma_{n, m}\left\|x_{n}-u_{n, m}\right\|\left\|A_{m} x_{n}-A_{m} p\right\| .
\end{aligned}
$$

This implies that

$$
\begin{aligned}
& \left(1-\alpha_{n}\right) k_{n} \gamma_{n, m}\left\|u_{n, m}-x_{n}\right\|^{2} \\
& \leq\left\|x_{n}-p\right\|^{2}-\left\|y_{n}-p\right\|^{2}+\left(1-\alpha_{n}\right)\left(k_{n}-1\right)\left\|x_{n}-p\right\|^{2} \\
& \quad+2 r_{n, m}\left(1-\alpha_{n}\right) k_{n} \sum_{m=1}^{N} \gamma_{n, m}\left\|x_{n}-u_{n, m}\right\|\left\|A_{m} x_{n}-A_{m} p\right\| \\
& \leq\left(\left\|x_{n}-p\right\|+\left\|y_{n}-p\right\|\right)\left\|x_{n}-y_{n}\right\|+\left(1-\alpha_{n}\right)\left(k_{n}-1\right)\left\|x_{n}-p\right\|^{2} \\
& \quad+2 r_{n, m}\left(1-\alpha_{n}\right) k_{n} \sum_{m=1}^{N} \gamma_{n, m}\left\|x_{n}-u_{n, m}\right\|\left\|A_{m} x_{n}-A_{m} p\right\| .
\end{aligned}
$$

In view of the restrictions (a) and (b), we obtain from (3.9) and (3.13) that

$$
\lim _{n \rightarrow \infty}\left\|u_{n, m}-x_{n}\right\|=0
$$

Since $\left\{x_{n}\right\}$ is bounded, there exists a subsequence $\left\{x_{n_{i}}\right\}$ of $\left\{x_{n}\right\}$ such that $x_{n_{i}} \rightarrow q$. Next, we show that $q \in F(S)$. Note that

$$
\left\|z_{n}-x_{n}\right\|=\left\|\sum_{m=1}^{N} \gamma_{n, m} u_{n, m}-x_{n}\right\| \leq \sum_{m=1}^{N} \gamma_{n, m}\left\|u_{n, m}-x_{n}\right\| .
$$

It follows from (3.14) that

$$
\lim _{n \rightarrow \infty}\left\|z_{n}-x_{n}\right\|=0 .
$$

On the other hand, we have

$$
\begin{aligned}
& \left\|x_{n}-\left(\beta_{n} x_{n}+\left(1-\beta_{n}\right) S^{n} x_{n}\right)\right\| \\
& \quad \leq\left\|\left(\beta_{n} x_{n}+\left(1-\beta_{n}\right) S^{n} x_{n}\right)-\left(\beta_{n} z_{n}+\left(1-\beta_{n}\right) S^{n} z_{n}\right)\right\|+\left\|\left(\beta_{n} z_{n}+\left(1-\beta_{n}\right) S^{n} z_{n}\right)-x_{n}\right\| \\
& \quad \leq \beta_{n}\left\|x_{n}-z_{n}\right\|+\left(1-\beta_{n}\right)\left\|S^{n} x_{n}-S^{n} z_{n}\right\|+\left\|\left(\beta_{n} z_{n}+\left(1-\beta_{n}\right) S^{n} z_{n}\right)-x_{n}\right\| \\
& \quad \leq \beta_{n}\left\|x_{n}-z_{n}\right\|+\left(1-\beta_{n}\right) L\left\|x_{n}-z_{n}\right\|+\left\|\left(\beta_{n} z_{n}+\left(1-\beta_{n}\right) S^{n} z_{n}\right)-x_{n}\right\| \\
& \quad \leq L\left\|x_{n}-z_{n}\right\|+\left\|\left(\beta_{n} z_{n}+\left(1-\beta_{n}\right) S^{n} z_{n}\right)-x_{n}\right\| .
\end{aligned}
$$


From (3.10) and (3.15), we find that

$$
\lim _{n \rightarrow \infty}\left\|x_{n}-\left(\beta_{n} x_{n}+\left(1-\beta_{n}\right) S^{n} x_{n}\right)\right\|=0
$$

Note that

$$
\begin{aligned}
\left\|S^{n} x_{n}-x_{n}\right\| & \leq\left\|S^{n} x_{n}-\left(\beta_{n} x_{n}+\left(1-\beta_{n}\right) S^{n} x_{n}\right)\right\|+\left\|\left(\beta_{n} x_{n}+\left(1-\beta_{n}\right) S^{n} x_{n}\right)-x_{n}\right\| \\
& \leq \beta_{n}\left\|S^{n} x_{n}-x_{n}\right\|+\left\|\left(\beta_{n} x_{n}+\left(1-\beta_{n}\right) S^{n} x_{n}\right)-x_{n}\right\|,
\end{aligned}
$$

which yields that

$$
\left(1-\beta_{n}\right)\left\|S^{n} x_{n}-x_{n}\right\| \leq\left\|\left(\beta_{n} x_{n}+\left(1-\beta_{n}\right) S^{n} x_{n}\right)-x_{n}\right\| .
$$

In view of the restriction (a), we find from (3.16) that

$$
\lim _{n \rightarrow \infty}\left\|S^{n} x_{n}-x_{n}\right\|=0
$$

Note that

$$
\begin{aligned}
\left\|x_{n}-S x_{n}\right\| \leq & \left\|x_{n}-x_{n+1}\right\|+\left\|x_{n+1}-S^{n+1} x_{n+1}\right\| \\
& +\left\|S^{n+1} x_{n+1}-S^{n+1} x_{n}\right\|+\left\|S^{n+1} x_{n}-S x_{n}\right\| \\
\leq & (1+L)\left\|x_{n}-x_{n+1}\right\|+\left\|x_{n+1}-S^{n+1} x_{n+1}\right\|+L\left\|S^{n} x_{n}-x_{n}\right\| .
\end{aligned}
$$

It follows from (3.7) and (3.17) that

$$
\lim _{n \rightarrow \infty}\left\|S x_{n}-x_{n}\right\|=0
$$

With the aid of Lemma 2.4, we find that $q \in F(S)$. Next, we prove $q \in \bigcap_{m=1}^{N} \operatorname{EP}\left(F_{m}\right)$. In view of (3.14), we find that $u_{n_{i}, m} \rightarrow q$ for each $1 \leq m \leq N$. From (3.14) and the restriction (b), we see that

$$
\lim _{n \rightarrow \infty} \frac{\left\|u_{n, m}-x_{n}\right\|}{r_{n, m}}=0, \quad \forall 1 \leq m \leq N
$$

Notice that

$$
F_{m}\left(u_{n, m}, u\right)+\left\langle A_{m} x_{n}, u-u_{n, m}\right\rangle+\frac{1}{r_{n, m}}\left\langle u-u_{n, m}, u_{n, m}-x_{n}\right\rangle \geq 0, \quad \forall u \in C .
$$

From (A2), we see that

$$
\left\langle A_{m} x_{n}, u-u_{n, m}\right\rangle+\frac{1}{r_{n, m}}\left\langle u-u_{n, m}, u_{n, m}-x_{n}\right\rangle \geq F_{m}\left(u, u_{n, m}\right) .
$$

Replacing $n$ by $n_{i}$, we arrive at

$$
\left\langle A_{m} x_{n_{i}}, u-u_{n_{i}, m}\right\rangle+\left\langle u-u_{n_{i}, m}, \frac{u_{n_{i}, m}-x_{n_{i}}}{r_{n_{i}, m}}\right\rangle \geq F_{m}\left(u, u_{n_{i}, m}\right) .
$$


For any $t$ with $0<t \leq 1$ and $u \in C$, let $\rho_{t}=t u+(1-t) q$. Since $u \in C$ and $q \in C$, we have $\rho_{t} \in C$. It follows from (3.19) that

$$
\begin{aligned}
\left\langle\rho_{t}\right. & \left.-u_{n_{i}, m}, A_{m} \rho_{t}\right\rangle \\
\geq & \left\langle\rho_{t}-u_{n_{i}, m}, A_{m} \rho_{t}\right\rangle-\left\langle A_{m} x_{n_{i}}, \rho_{t}-u_{n_{i}, m}\right\rangle-\left\langle\rho_{t}-u_{n_{i}, m}, \frac{u_{n_{i}, m}-x_{n_{i}}}{r_{n_{i}, m}}\right\rangle+F_{m}\left(\rho_{t}, u_{n_{i}, m}\right) \\
= & \left\langle\rho_{t}-u_{n_{i}, m}, A_{m} \rho_{t}-A_{m} u_{n, i, m}\right\rangle+\left\langle\rho_{t}-u_{n_{i}, m}, A_{m} u_{n, i, m}-A_{m} x_{n_{i}}\right\rangle \\
& -\left\langle\rho_{t}-u_{n_{i}, m}, \frac{u_{n_{i}, m}-x_{n_{i}}}{r_{n_{i}, m}}\right\rangle+F\left(\rho_{t}, u_{n_{i}, m}\right) .
\end{aligned}
$$

Since $A_{m}$ is Lipschitz continuous, we find from (3.14) that $A_{m} u_{n, i, m}-A_{m} x_{n_{i}} \rightarrow 0$ as $i \rightarrow \infty$. From the monotonicity of $A_{m}$, we get that

$$
\left\langle\rho_{t}-u_{n_{i}, m}, A_{m} \rho_{t}-A_{m} u_{n, i, m}\right\rangle \geq 0 .
$$

In view of (A4), we find from (3.20) that

$$
\left\langle\rho_{t}-q, A_{m} \rho_{t}\right\rangle \geq F\left(\rho_{t}, q\right) \text {. }
$$

With the aid of (A1), (A4), we obtain from (3.21) that

$$
\begin{aligned}
0 & =F\left(\rho_{t}, \rho_{t}\right) \leq t F\left(\rho_{t}, u\right)+(1-t) F\left(\rho_{t}, q\right) \\
& \leq t F\left(\rho_{t}, u\right)+(1-t)\left\langle\rho_{t}-q, A_{m} \rho_{t}\right\rangle \\
& =t F\left(\rho_{t}, u\right)+(1-t) t\left\langle u-q, A_{m} \rho_{t}\right\rangle
\end{aligned}
$$

which implies that

$$
F\left(\rho_{t}, u\right)+(1-t)\left\langle u-q, A_{m} \rho_{t}\right\rangle \geq 0 .
$$

Letting $t \rightarrow 0$ in the above inequality, we arrive at

$$
F(q, u)+\left\langle u-q, A_{m} q\right\rangle \geq 0 .
$$

This shows that $q \in \operatorname{EP}\left(F_{m}, A_{m}\right), \forall 1 \leq m \leq N$. This completes the proof that $q \in \mathcal{F}$. Put $\bar{x}=P_{\mathcal{F}} x_{1}$, we obtain that

$$
\begin{aligned}
\left\|x_{1}-\bar{x}\right\| & \leq\left\|x_{1}-q\right\| \leq \liminf _{i \rightarrow \infty}\left\|x_{1}-x_{n_{i}}\right\| \\
& \leq \limsup _{i \rightarrow \infty}\left\|x_{1}-x_{n_{i}}\right\| \leq\left\|x_{1}-\bar{x}\right\|,
\end{aligned}
$$

which yields that

$$
\lim _{i \rightarrow \infty}\left\|x_{1}-x_{n_{i}}\right\|=\left\|x_{1}-q\right\|=\left\|x_{1}-\bar{x}\right\| .
$$

It follows that $\left\{x_{n_{i}}\right\}$ converges strongly to $\bar{x}$. Therefore, we can conclude that the sequence $\left\{x_{n}\right\}$ converges strongly to $\bar{x}=P_{\mathcal{F}} x_{1}$. This completes the proof. 
Based on Theorem 3.1, we have the following results.

Corollary 3.2 Let $C$ be a nonempty closed convex subset of a real Hilbert space H. Let $F$ be a bifunction from $C \times C$ to $\mathbb{R}$ which satisfies (A1)-(A4) and let $A: C \rightarrow H$ be a $\xi$-inversestrongly monotone mapping. Let $S: C \rightarrow C$ be an asymptotically $\kappa$-strict pseudocontraction. Assume that $\mathcal{F}:=F(S) \cap \mathrm{EP}(F, A)$ is nonempty and bounded. Let $\left\{\alpha_{n}\right\}$ and $\left\{\beta_{n}\right\}$ be sequences in $[0,1]$ and $\left\{r_{n}\right\}$ be a positive sequence. Let $\left\{x_{n}\right\}$ be a sequence generated in the following manner:

$$
\left\{\begin{array}{l}
x_{1} \in H, \\
C_{1}=H, \\
F\left(u_{n}, u\right)+\left\langle A x_{n}, u-u_{n}\right\rangle+\frac{1}{r_{n}}\left\langle u-u_{n}, u_{n}-x_{n}\right\rangle \geq 0, \quad \forall u \in C, \\
y_{n}=\alpha_{n} x_{n}+\left(1-\alpha_{n}\right)\left(\beta_{n} u_{n}+\left(1-\beta_{n}\right) S^{n} u_{n}\right), \\
C_{n+1}=\left\{w \in C_{n}:\left\|y_{n}-w\right\|^{2} \leq\left\|x_{n}-w\right\|^{2}+\theta_{n}\right\}, \\
x_{n+1}=P_{C_{n+1}} x_{1}, \quad n \geq 0,
\end{array}\right.
$$

where $\theta_{n}=\left(1-\alpha_{n}\right)\left(k_{n}-1\right) \Theta_{n}$ and $\Theta_{n}=\sup \left\{\left\|x_{n}-w\right\|^{2}: w \in \mathcal{F}\right\}$. Assume that the control sequences $\left\{\alpha_{n}\right\},\left\{\beta_{n}\right\}$ and $\left\{r_{n}\right\}$ satisfy the following restrictions:

(a) $0 \leq \alpha_{n} \leq a<1,0<\kappa \leq \beta_{n} \leq b<1$;

(b) $0<d \leq r_{n} \leq e<2 \xi$.

Then the sequence $\left\{x_{n}\right\}$ converges strongly to some point $\bar{x}$, where $\bar{x}=P_{\mathcal{F}} x_{1}$.

Proof Putting $F_{m} \equiv F, A_{m} \equiv A$ and $r_{n, m} \equiv r_{n}$ in Theorem 3.1, we see that $\sum_{m=1}^{N} \gamma_{n, m} u_{n, m} \equiv$ $u_{n}$. With the aid of Theorem 3.1, we can easily conclude the desired conclusion.

If $S$ is asymptotically nonexpansive, then Corollary 3.2 is reduced to the following.

Corollary 3.3 Let C be a nonempty closed convex subset of a real Hilbert space H. Let $F$ be a bifunction from $C \times C$ to $\mathbb{R}$ which satisfies (A1)-(A4) and let $A: C \rightarrow H$ be a $\xi$-inversestrongly monotone mapping. Let $S: C \rightarrow C$ be an asymptotically nonexpansive mapping. Assume that $\mathcal{F}:=F(S) \cap \operatorname{EP}(F, A)$ is nonempty and bounded. Let $\left\{\alpha_{n}\right\}$ and $\left\{\beta_{n}\right\}$ be sequences in $[0,1]$ and let $\left\{r_{n}\right\}$ be a positive sequence. Let $\left\{x_{n}\right\}$ be a sequence generated in the following manner:

$$
\left\{\begin{array}{l}
x_{1} \in H \\
C_{1}=H, \\
F\left(u_{n}, u\right)+\left\langle A x_{n}, u-u_{n}\right\rangle+\frac{1}{r_{n}}\left\langle u-u_{n}, u_{n}-x_{n}\right\rangle \geq 0, \quad \forall u \in C, \\
y_{n}=\alpha_{n} x_{n}+\left(1-\alpha_{n}\right)\left(\beta_{n} u_{n}+\left(1-\beta_{n}\right) S^{n} u_{n}\right), \\
C_{n+1}=\left\{w \in C_{n}:\left\|y_{n}-w\right\|^{2} \leq\left\|x_{n}-w\right\|^{2}+\theta_{n}\right\}, \\
x_{n+1}=P_{C_{n+1}} x_{1}, \quad n \geq 0
\end{array}\right.
$$

where $\theta_{n}=\left(1-\alpha_{n}\right)\left(k_{n}-1\right) \Theta_{n}$ and $\Theta_{n}=\sup \left\{\left\|x_{n}-w\right\|^{2}: w \in \mathcal{F}\right\}$. Assume that the control sequences $\left\{\alpha_{n}\right\},\left\{\beta_{n}\right\}$ and $\left\{r_{n}\right\}$ satisfy the following restrictions:

(a) $0 \leq \alpha_{n} \leq a<1,0 \leq \beta_{n} \leq b<1$;

(b) $0<d \leq r_{n} \leq e<2 \xi$.

Then the sequence $\left\{x_{n}\right\}$ converges strongly to some point $\bar{x}$, where $\bar{x}=P_{\mathcal{F}} x_{1}$. 
Putting $\beta_{n} \equiv 0$ in Corollary 3.3, we have the following.

Corollary 3.4 Let $C$ be a nonempty closed convex subset of a real Hilbert space H. Let $F$ be a bifunction from $C \times C$ to $\mathbb{R}$ which satisfies (A1)-(A4) and let $A: C \rightarrow H$ be a $\xi$-inversestrongly monotone mapping. Let $S: C \rightarrow C$ be an asymptotically nonexpansive mapping. Assume that $\mathcal{F}:=F(S) \cap \operatorname{EP}(F, A)$ is nonempty and bounded. Let $\left\{\alpha_{n}\right\}$ be a sequence in $[0,1]$ and $\left\{r_{n}\right\}$ be a positive sequence. Let $\left\{x_{n}\right\}$ be a sequence generated in the following manner:

$$
\left\{\begin{array}{l}
x_{1} \in H \\
C_{1}=H \\
F\left(u_{n}, u\right)+\left\langle A x_{n}, u-u_{n}\right\rangle+\frac{1}{r_{n}}\left\langle u-u_{n}, u_{n}-x_{n}\right\rangle \geq 0, \quad \forall u \in C \\
y_{n}=\alpha_{n} x_{n}+\left(1-\alpha_{n}\right) S^{n} u_{n} \\
C_{n+1}=\left\{w \in C_{n}:\left\|y_{n}-w\right\|^{2} \leq\left\|x_{n}-w\right\|^{2}+\theta_{n}\right\} \\
x_{n+1}=P_{C_{n+1}} x_{1}, \quad n \geq 0
\end{array}\right.
$$

where $\theta_{n}=\left(1-\alpha_{n}\right)\left(k_{n}-1\right) \Theta_{n}$ and $\Theta_{n}=\sup \left\{\left\|x_{n}-w\right\|^{2}: w \in \mathcal{F}\right\}$. Assume that the control sequences $\left\{\alpha_{n}\right\}$ and $\left\{r_{n}\right\}$ satisfy the following restrictions:

(a) $0 \leq \alpha_{n} \leq a<1$;

(b) $0<d \leq r_{n} \leq e<2 \xi$.

Then the sequence $\left\{x_{n}\right\}$ converges strongly to some point $\bar{x}$, where $\bar{x}=P_{\mathcal{F}} x_{1}$.

Corollary 3.5 Let $C$ be a nonempty closed convex subset of a real Hilbert space H. Let $A_{m}: C \rightarrow H$ be a $\xi_{m}$-inverse-strongly monotone mapping, for each $1 \leq m \leq N$, where $N \geq 1$ is some positive integer. Let $S: C \rightarrow C$ be an asymptotically $\kappa$-strict pseudocontraction. Assume that $\mathcal{F}:=F(S) \cap \bigcap_{m=1}^{N} \operatorname{VI}\left(C, A_{m}\right)$ is nonempty and bounded. Let $\left\{\alpha_{n}\right\}$ and $\left\{\beta_{n}\right\}$ be sequences in $[0,1]$ and $\left\{r_{n, m}\right\}$ be a positive sequence. Let $\left\{\gamma_{n, m}\right\}$ be a sequence in $[0,1]$ for each $1 \leq m \leq N$ such that $\sum_{m=1}^{N} \gamma_{n, m}=1$. Let $\left\{x_{n}\right\}$ be a sequence generated in the following manner:

$$
\left\{\begin{array}{l}
x_{1} \in H, \\
C_{1}=H, \\
u_{n, m}=P_{C}\left(x_{n}-r_{n, m} A_{m} x_{n}\right), \\
y_{n}=\alpha_{n} x_{n}+\left(1-\alpha_{n}\right)\left(\beta_{n} \sum_{m=1}^{N} \gamma_{n, m} u_{n, m}+\left(1-\beta_{n}\right) S^{n} \sum_{m=1}^{N} \gamma_{n, m} u_{n, m}\right), \\
C_{n+1}=\left\{w \in C_{n}:\left\|y_{n}-w\right\|^{2} \leq\left\|x_{n}-w\right\|^{2}+\theta_{n}\right\}, \\
x_{n+1}=P_{C_{n+1}} x_{1}, \quad n \geq 0,
\end{array}\right.
$$

where $\theta_{n}=\left(1-\alpha_{n}\right)\left(k_{n}-1\right) \Theta_{n}$ and $\Theta_{n}=\sup \left\{\left\|x_{n}-w\right\|^{2}: w \in \mathcal{F}\right\}$. Assume that the control sequences $\left\{\alpha_{n}\right\},\left\{\beta_{n}\right\},\left\{\gamma_{n, 1}\right\}, \ldots,\left\{\gamma_{n, N}\right\},\left\{r_{n, 1}\right\}, \ldots$ and $\left\{r_{n, N}\right\}$ satisfy the following restrictions:

(a) $0 \leq \alpha_{n} \leq a<1, \kappa \leq \beta_{n} \leq b<1$;

(b) $0<c \leq \gamma_{n, m} \leq 1$ and $0<d \leq r_{n, m} \leq e<2 \xi_{m}$ for each $1 \leq m \leq N$.

Then the sequence $\left\{x_{n}\right\}$ converges strongly to some point $\bar{x}$, where $\bar{x}=P_{\mathcal{F}} x_{1}$.

Proof In Theorem 3.1, put $F_{m}(x, y)=0$ for all $x, y \in C$. From

$$
\left\langle A_{m} x_{n}, u-u_{n, m}\right\rangle+\frac{1}{r_{n, m}}\left\langle u-u_{n, m}, u_{n, m}-x_{n}\right\rangle,
$$


we have

$$
\left\langle u-u_{n, m}, x_{n}-u_{n, m}-r_{n, m} A_{m} x_{n}\right\rangle \geq 0, \quad \forall u \in C .
$$

This implies that

$$
u_{n, m}=P_{C}\left(x_{n}-r_{n, m} A_{m} x_{n}\right)
$$

In view of Theorem 3.1, we can immediately obtain the desired conclusion. This completes the proof.

Corollary 3.6 Let $C$ be a nonempty closed convex subset of a real Hilbert space H. Let $F_{m}$ be a bifunction from $C \times C$ to $\mathbb{R}$ which satisfies (A1)-(A4) for each $1 \leq m \leq N$, where $N \geq 1$ is some positive integer. Let $S: C \rightarrow C$ be an asymptotically $\kappa$-strict pseudocontraction. Assume that $\mathcal{F}:=F(S) \cap \bigcap_{m=1}^{N} \operatorname{EP}\left(F_{m}\right)$ is nonempty and bounded. Let $\left\{\alpha_{n}\right\}$ and $\left\{\beta_{n}\right\}$ be sequences in $[0,1]$ and $\left\{r_{n, m}\right\}$ be a positive sequence. Let $\left\{\gamma_{n, m}\right\}$ be a sequence in $[0,1]$ for each $1 \leq m \leq N$ such that $\sum_{m=1}^{N} \gamma_{n, m}=1$. Let $\left\{x_{n}\right\}$ be a sequence generated in the following manner:

$$
\left\{\begin{array}{l}
x_{1} \in H, \\
C_{1}=H, \\
F_{m}\left(u_{n, m}, u\right)+\frac{1}{r_{n, m}}\left\langle u-u_{n, m}, u_{n, m}-x_{n}\right\rangle \geq 0, \quad \forall u \in C, \\
y_{n}=\alpha_{n} x_{n}+\left(1-\alpha_{n}\right)\left(\beta_{n} \sum_{m=1}^{N} \gamma_{n, m} u_{n, m}+\left(1-\beta_{n}\right) S^{n} \sum_{m=1}^{N} \gamma_{n, m} u_{n, m}\right), \\
C_{n+1}=\left\{w \in C_{n}:\left\|y_{n}-w\right\|^{2} \leq\left\|x_{n}-w\right\|^{2}+\theta_{n}\right\}, \\
x_{n+1}=P_{C_{n+1}} x_{1}, \quad n \geq 0,
\end{array}\right.
$$

where $\theta_{n}=\left(1-\alpha_{n}\right)\left(k_{n}-1\right) \Theta_{n}$ and $\Theta_{n}=\sup \left\{\left\|x_{n}-w\right\|^{2}: w \in \mathcal{F}\right\}$. Assume that the control sequences $\left\{\alpha_{n}\right\},\left\{\beta_{n}\right\},\left\{\gamma_{n, 1}\right\}, \ldots,\left\{\gamma_{n, N}\right\},\left\{r_{n, 1}\right\}, \ldots$ and $\left\{r_{n, N}\right\}$ satisfy the following restrictions:

(a) $0 \leq \alpha_{n} \leq a<1, \kappa \leq \beta_{n} \leq b<1$

(b) $0<c \leq \gamma_{n, m} \leq 1$ and $0<d \leq r_{n, m} \leq e<\infty$ for each $1 \leq m \leq N$.

Then the sequence $\left\{x_{n}\right\}$ converges strongly to some point $\bar{x}$, where $\bar{x}=P_{\mathcal{F}} x_{1}$.

Proof In Theorem 3.1, put $A_{m}=0$. Then, for any $\xi_{m}>0$, we see that

$$
\left\langle x-y, A_{m} x-A_{m} y\right\rangle \geq \xi_{m}\left\|A_{m} x-A_{m} y\right\|^{2}, \quad \forall x, y \in C .
$$

Let $\left\{r_{n, m}\right\}$ be a sequence satisfying the restriction $d \leq r_{n} \leq e$, where $d, e \in(0, \infty)$. Then we can obtain the desired conclusion easily from Theorem 3.1. This completes the proof. 


\section{Author details}

'School of Mathematics and Information Science, North China University of Water Resources and Electric Power,

Zhengzhou, China. ${ }^{2}$ College of Science, Hebei University of Engineering, Handan, 056038, China.

\section{Acknowledgements}

The authors are grateful to the referees for their valuable comments and suggestions which improved the contents of the article.

\section{Received: 17 March 2013 Accepted: 30 April 2013 Published: 17 May 2013}

\section{References}

1. liduka, H: Fixed point optimization algorithm and its application to network bandwidth allocation. J. Comput. Appl. Math. 236, 1733-1742 (2012)

2. Mahato, NK, Nahak, C: Equilibrium problem under various types of convexities in Banach space. J. Math. Comput. Sci. 1, 77-88 (2011)

3. Shen, J, Pang, LP: An approximate bundle method for solving variational inequalities. Commun. Optim. Theory 1 , 1-18 (2012)

4. Cho, SY, Kang, SM: Approximation of common solutions of variational inequalities via strict pseudocontractions. Acta Math. Sci. 32, 1607-1618 (2012)

5. Abdel-Salam, HS, Al-Khaled, K: Variational iteration method for solving optimization problems. J. Math. Comput. Sci. 2, 1475-1497 (2012)

6. Censor, Y, Cohen, N, Kutscher, T, Shamir, J: Summed squared distance error reduction by simultaneous multiprojections and applications. Appl. Math. Comput. 126, 157-179 (2002)

7. Carmi, A, Censor, Y, Gurfil, P: Convex feasibility modeling and projection methods for sparse signal recovery. J. Comput. Appl. Math. 236, 4318-4335 (2012)

8. Qin, X, Chang, SS, Kang, SM: Iterative methods for generalized equilibrium problems and fixed point problems with applications. Nonlinear Anal. 11, 2963-2972 (2010)

9. Khader, MM, Mohamed, ST: Numerical treatment for first order neutral delay differential equations using spline functions. Eng. Math. Lett. 1, 32-43 (2012)

10. Noor, MA, Noor, KI, Waseem, M: Decomposition method for solving system of linear equations. Eng. Math. Lett. 2 34-41 (2012)

11. Kim, JK, Anh, PN, Nam, YM: Strong convergence of an extended extragradient method for equilibrium problems and fixed point problems. J. Korean Math. Soc. 49, 187-200 (2012)

12. Cho, SY, Kang, SM: Approximation of fixed points of pseudocontraction semigroups based on a viscosity iterative process. Appl. Math. Lett. 24, 224-228 (2011)

13. Haugazeau, Y: Sur les inéquations variationnelles et la minimisation de fonctionnelles convexes. Thése, Université de Paris, Paris, France (1968)

14. Goebel, K, Kirk, WA: A fixed point theorem for asymptotically nonexpansive mappings. Proc. Am. Math. Soc. 35 171-174 (1972)

15. Browder, FE, Petryshyn, WV: Construction of fixed points of nonlinear mappings in Hilbert space. J. Math. Anal. Appl. 20, 197-228 (1967)

16. Qihou, L: Convergence theorems of the sequence of iterates for asymptotically demicontractive and hemicontractive mappings. Nonlinear Anal. 26, 1835-1842 (1996)

17. Schu, J: Iterative construction of fixed points of asymptotically nonexpansive mappings. J. Math. Anal. Appl. 158, 407-413 (1991)

18. Qin, X, Shang, M, Su, Y: Strong convergence of a general iterative algorithm for equilibrium problems and variational inequality problems. Math. Comput. Model. 48, 1033-1046 (2008)

19. Ye, J, Huang, J: Strong convergence theorems for fixed point problems and generalized equilibrium problems of three relatively quasi-nonexpansive mappings in Banach spaces. J. Math. Comput. Sci. 1, 1-18 (2011)

20. Qin, X, Cho, SY, Kang, SM: On hybrid projection methods for asymptotically quasi- $\boldsymbol{\phi}$-nonexpansive mappings. Appl. Math. Comput. 215, 3874-3883 (2010)

21. Zegeye, $\mathrm{H}$, Shahzad, N: Strong convergence theorem for a common point of solution of variational inequality and fixed point problem. Adv. Fixed Point Theory 2, 374-397 (2012)

22. Yuan, Q, Shang, M: Convergence of an extragradient-like iterative algorithm for monotone mappings and nonexpansive mappings. Fixed Point Theory Appl. 2013, 67 (2013)

23. Kang, SM, Cho, SY, Liu, Z: Convergence of iterative sequences for generalized equilibrium problems involving inverse-strongly monotone mappings. J. Inequal. Appl. 2010, 827082 (2010)

24. Qin, X, Cho, YJ, Kang, SM: Convergence theorems of common elements for equilibrium problems and fixed point problems in Banach spaces. J. Comput. Appl. Math. 225, 20-30 (2009)

25. Tada, A, Takahashi, W: Weak and strong convergence theorem for an equilibrium problem and a nonexpansive mapping. J. Optim. Theory Appl. 133, 359-370 (2007)

26. Qin, X, Cho, SY, Kang, SM: An extragradient-type method for generalized equilibrium problems involving strictly pseudocontractive mappings. J. Glob. Optim. 49, 679-693 (2011)

27. Kim, JK: Strong convergence theorems by hybrid projection methods for equilibrium problems and fixed point problems of the asymptotically quasi- $\boldsymbol{\phi}$-nonexpansive mappings. Fixed Point Theory Appl. 2011, 10 (2011)

28. Kim, JK, Cho, SY, Qin, X: Hybrid projection algorithms for generalized equilibrium problems and strictly pseudocontractive mappings. J. Inequal. Appl. 2010, 312602 (2010)

29. Yang, L, Zhao, F, Kim, JK: Hybrid projection method for generalized mixed equilibrium problem and fixed point problem of infinite family of asymptotically quasi- $\boldsymbol{\phi}$-nonexpansive mappings in Banach spaces. Appl. Math. Comput. 218, 6072-6082 (2012)

30. Qin, X, Cho, SY, Kang, SM: Strong convergence of shrinking projection methods for quasi- $\boldsymbol{\phi}$-nonexpansive mappings and equilibrium problems. J. Comput. Appl. Math. 234, 750-760 (2010) 
31. Blum, E, Oettli, W: From optimization and variational inequalities to equilibrium problems. Math. Stud. 63, 123-145 (1994)

32. Reinermann, J: Uber fixpunkte kontrahievuder Abbidungen und schwach konvergente Tooplite-Verfahren. Arch. Math. 20, 59-64 (1969)

33. Sahu, DR, Xu, HK, Yao, JC: Asymptotically strict pseudocontractive mappings in the intermediate sense. Nonlinear Anal. 70, 3502-3511 (2009)

doi:10.1186/1029-242X-2013-251

Cite this article as: Cheng and Wu: On asymptotically strict pseudocontractions and equilibrium problems. Journal of Inequalities and Applications 2013 2013:251.

Submit your manuscript to a SpringerOpen ${ }^{\circ}$ journal and benefit from:

- Convenient online submission

- Rigorous peer review

- Immediate publication on acceptance

- Open access: articles freely available online

- High visibility within the field

- Retaining the copyright to your article

Submit your next manuscript at $>$ springeropen.com 